\title{
Correlated disorder-to-order crossover in the local structure of $\mathrm{K}_{\mathrm{x}} \mathrm{Fe}_{2-\mathrm{y}} \mathrm{Se}_{2-\mathrm{z}} \mathrm{S}_{\mathrm{z}}$ superconductor
}

\author{
E.S. Bozin ${ }^{1}$, R.J. Koch ${ }^{1}$, P. Mangelis ${ }^{2}$, H.C. Lei ${ }^{1}$, R. Neder ${ }^{3}$, M. McDonnell ${ }^{4}$, M. Feygenson ${ }^{4}$, C. Petrovic ${ }^{1}$, A. Lappas ${ }^{2}$ \\ ${ }^{1}$ Brookhaven National Laboratory, Upton, NY, USA, \\ ${ }^{2}$ IESL FORTH, Heraklion, Greece, \\ ${ }^{3}$ University of Erlangen-Nuremberg, Erlangen, Germany, \\ ${ }^{4}$ Oak Ridge National Laboratory, Oak Ridge, TN, United States \\ bozin@bnl.gov
}

A detailed account of the local atomic structure and disorder at $5 \mathrm{~K}$ across the phase diagram of the high-temperature superconductor $\mathrm{K}_{\mathrm{x}} \mathrm{Fe}_{2-\mathrm{y}} \mathrm{Se}_{2-\mathrm{z}} \mathrm{S}_{\mathrm{z}}(0 \leq \mathrm{z} \leq 2)$ is obtained from neutron total scattering and associated atomic pair distribution function (PDF) approaches [1]. Various model-independent and model-dependent aspects of the analysis reveal a high level of structural complexity on the nanometer length scale. Evidence is found for considerable disorder in the c-axis stacking of the $\mathrm{FeSe}_{1-\mathrm{x}} \mathrm{S}_{\mathrm{x}}$ slabs without observable signs of turbostratic character of the disorder. In contrast to the related $\mathrm{FeCh}(\mathrm{Ch}=\mathrm{S}$, Se)-type superconductors, substantial Fe-vacancies are present in $\mathrm{K}_{\mathrm{x}} \mathrm{Fe}_{2-\mathrm{y}} \mathrm{Se}_{2-\mathrm{z}} \mathrm{S}_{\mathrm{z}}$, deemed detrimental for superconductivity when ordered. Our study suggests that the distribution of vacancies significantly modifies the iron-chalcogen bond-length distribution, in agreement with observed evolution of the PDF signal. A crossover-like transition is observed at a composition of $\mathrm{z} \approx 1$, from a correlated disorder state at the selenium end to a more vacancy-ordered (VO) state closer to the sulfur end of the phase diagram. The S-content-dependent measures of the local structure are found to exhibit distinct behavior on either side of this crossover, correlating well with the evolution of the superconducting state to that of a magnetic semiconductor toward the $\mathrm{z} \approx 2$ end. The behavior reinforces the idea of the intimate relationship of correlated $\mathrm{Fe}$ vacancy order in the local structure and the emergent electronic properties.

[1] P. Mangelis et al., (2019) Physical Review B 100, 094108.

Keywords: iron-based superconductors, local atomic structure, correlated disorder, total scattering, neutron scattering

Work at Brookhaven National Laboratory was supported by the U.S. Department of Energy, Office of Science, Office of Basic Energy Sciences (DOE-BES) under Contract No. DE-SC0012704. Alexandros Lappas acknowledges support by the U.S. Office of Naval Research Global, NICOP Grant Award No. N62909-17-1-2126. This research used resources at the Spallation Neutron Source, a U.S. Department of Energy Office of Science User Facility operated by the Oak Ridge National Laboratory 